\title{
Flame Radiation Distribution from Fires
}

\author{
MICHAEL A. DELICHATSIOS \\ Factory Mutual Research Corporation \\ Norwood, MA 02062 \\ PATRICIA TATEM \\ Naval Research Laboratory \\ Washington D.C. 20375 \\ YONGGANG CHEN and VAHID MOTEVALLI \\ Worcester Polytechnic Institute \\ Worcester, MA 01609
}

\begin{abstract}
A methodology and a model are presented for the calculation of radiation distribution from fires. Extensive application of the model is discussed with regard to turbulent buoyant jet fires wherein radiation is controlled by soot and the flame size is such that it produces optically thin conditions. The local soot concentration is modelled, according to previous results, to be 1) proportional to the inverse smoke-point heat release rate, $Y_{s} \sim 1 / \dot{Q}_{\text {sp }}$ and 2) a function of the local mixture fraction and temperature. The local volumetric emissive power is averaged over the turbulent fluctuations to obtain the radiation losses for optically thin flame situations. Combustion is modelled, in a standard way, by an ensemble of laminar flamelets whose chemical composition of species is known from state relationships. The combustion model is an integral model that has been validated by extensive data from turbulent buoyant jet flames whereas the radiation model is validated here by comparison with propane turbulent jet flames; this comparison allows the determination of a proportionality coefficient for the soot concentration and radiation term. The generality and soundness of the model has been determined by applying it to predict the jet flame radiation from another fuel, propylene (while maintaining the same proportionality constant for the soot as for the propane). Preliminary results and favorable comparisons with experiments are also shown for pool fires of diameter up to $1 \mathrm{~m}$. The proposed methodology and the model can be applied to an arbitrary burning material whose laminar smoke-point heat release rate has been measured in laboratory experiments.
\end{abstract}

KEYWORDS: radiation, diffusion flame, pool fire radiation

\section{INTRODUCTION}

For the prediction of fire growth in compartments, one needs predictions of heat fluxes and products of combustion originating from the burning of an arbitrary material, whose appropriate burning properties can be deduced from flammability test measurements. Given the complexity of the fire growth problem in a compartment, detailed simulation of 
the combustion process (i.e., full Navier-Stokes or $\mathbf{k - \epsilon - g}$ equations plus combustion, not yet available) is impractical for an arbitrary buming material. Moreover, application of simple correlations (which are currently used in compartment fire models) can not adequately capture the important effects of geometry, flame radiation distribution and vitiation. Thus, an integral combustion model $[1,2]$ has been considered a desirable tool for predicting flame characteristics (e.g. flame height, radiation loss) as well as major species concentrations (e.g., $\mathrm{H}_{2} \mathrm{O}, \mathrm{CO}_{2}, \mathrm{CO}$ ) for an arbitrary burning material (e.g., non-charring and charring). Development of an integral combustion model requires complete understanding of the physics together with a comprehensive comparison with experimental data.

In this paper, we focus on flame radiation from soot which is usually the main source of radiation in fires. The following criteria are used for developing a model for radiation: 1) the model should be applicable for all ranges of optical thickness (from optically thin to optically thick flames); 2) the model should be applicable for fire originating from any flammable material by using its flammability properties. For soot radiation, especially, one would utilize, for example, the laminar smoke-point heat release rate, $\dot{Q}_{\mathrm{sp}}$, to predict local soot concentrations[3]; 3) the model could be applicable to wall, pool and jet fires; 4) the model could incorporate vitiation effects.

\section{MODEL DESCRIPTION AND ENERGY BALANCE}

The integral combustion model presented earlier[4] has been developed for turbulent jet diffusion flames and includes, as its most important component, flame radiation and its relation to flame turbulence and the sooting tendency of the fuel. The buoyant jet is modelled using integral conservation equations and an ensemble of flamelets to model reactions and combustion. The energy equation is simultaneously solved with the flow equations, accounting for radiation losses and employs a simple soot formation model. The model computes the radiant losses, the incompleteness of combustion and the CO-yield by using the following physics:1) the lower part of the flame is modelled by diffusion flamelets using a probability distribution function and 2) the upper part, as the flame cools due to radiant losses, is modelled as a premixed mixture sustaining $\mathrm{CO}+$ soot oxidation (Figure 1). The main characteristics of the integral model are the following:

1) Instead of an entrainment equation, a dimensionless correlation for entrainment spanning the range from momentum to buoyant turbulent jet flames has been employed and validated[4].

2) A direct relationship for the fluctuation of a conserved scalar, $\xi$, inspired by recent work[5] and validated by recent experiments is also used[6]. The relationship is:

$$
\frac{\bar{\xi}^{\prime 2}}{a \bar{\xi}\left(b \bar{\xi}_{c}-\bar{\xi}\right)}=\frac{1}{1+c \frac{a\left(b \bar{\xi}_{c}-\bar{\xi}\right)}{1-\bar{\xi}}}
$$

wherein a represents molecular mixing, b represents intermittency, c represents initial condition and are determined from experiments[5,6]. In equation (1a), $\bar{\xi}_{\mathrm{c}}$ and $\bar{\xi}$ are time averaged centerline and local mixture fractions, respectively. The constants in eqn. (1a) have the following values: $a=4.5, b=1.4, c=1$. Equation (1a) is also consistent with $a$ 
conservation equation for fluctuation of a conserved scalar. One should notice that the level of fluctuations in turbulent jet flames $\bar{\xi}^{\prime} / \bar{\xi}$ can exceed one[6]. A $\beta$-PDF (Probability Distribution Function) for the conserved scalar[1] is obtained using eqn. (1a).

3) In the present model, radial profiles for the mixture fraction and velocity are used in order to preserve the strong nonlinearity of the combustion process[1], i.e.

$$
\begin{aligned}
& \bar{\xi}=\bar{\xi}_{c}\left(1-\frac{\psi}{\psi_{f}}\right) \\
& \bar{u}=\bar{u}_{c}\left(1-\frac{\psi}{\psi_{t}}\right)
\end{aligned}
$$

where $\vec{u}$ and $\vec{u}_{c}$ are the mean local and centerline velocities while $\Psi$ and $\Psi_{t}$ are the stream function and entrainment rate, respectively[1].

4) Most importantly, a flame radiation model applicable for luminous (soot radiation) and optically thin flame is presented whose sooting

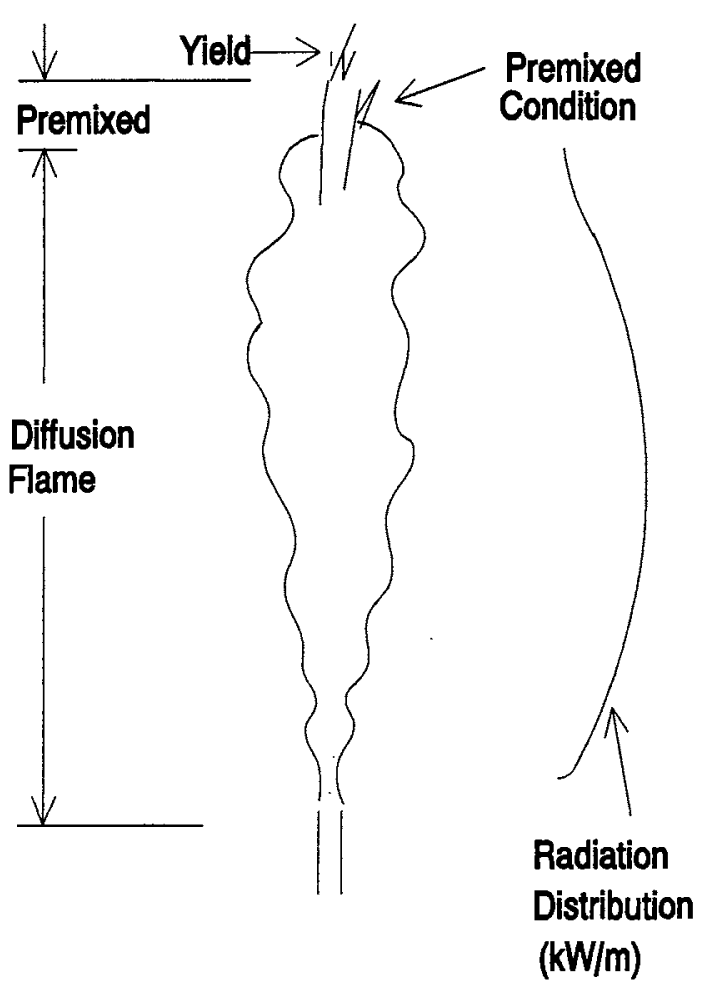

Figure 1 Combustion Regions for a turbulent buoyant jet diffusion flame tendency is characterized by the laminar smoke-point height.

The soot radiation model is founded on a phenomenological description for soot formation which has been established from data in laminar diffusion flames[7]; the soot concentration can be determined by the following rate equation[7]:

$$
\frac{d Y_{s}}{d t}=A \rho^{2} \frac{\xi-\xi_{s}}{1-\xi_{s}} e^{-E / R T}
$$

wherein $\xi, \xi_{3}$ are the fuel mixture fraction and its stoichiometric value, $E$ is the activation energy for soot formation and $\mathrm{T}$ is the absolute temperature. As an approximation the destruction of soot can be ignored. The proportionality factor A which includes the fuel structure chemistry effects, is proportional to the inverse of the laminar smoke-point heat release rate[7]: 
The proportionality constant is properly defined in ref 7 .

Prior to applying eqn. (1b) to turbulent flows, it is useful to know what is required for modelling radiation losses in an optically thin medium. The flame radiation loss due to soot can be treated in this case as an volumetric emission described by an expression which can be reduced to the following form[7]:

$$
\dot{q}_{s}^{\prime \prime \prime}=3.26 \cdot 10^{-4} \frac{P}{R p_{s}} Y T^{4} W / m^{3} K^{5}
$$

where $P$ is the pressure, $R$ is the gas constant and $\rho_{b}$ is the soot density.

For a turbulent flow, one has to average the volumetric radiant loss (eqn. (2)) over the turbulent fluctuating field. Local instantaneous values of the temperature, $\mathrm{T}$, and the soot concentration, $Y_{n}$, needed for this purpose, are obtained by using the representation of turbulent combustion by an ensemble of flamelets that may also be subjected to a spectrum of turbulent straining rates.

Following such modelling, the instantaneous laminar flamelet temperature, $\mathrm{T}_{\mathrm{L}}$, would be a function of the mixture fraction, $\xi$, as it is customarily done in flamelet models (a modification to this relationship will be presented later in this work to account for the radiant losses). The soot concentration can be obtained by integration of the soot formation rate (eqn. (1b)) over the local flow field of a strained diffusion flame[7]. The soot concentration becomes:

$$
Y_{s}=\frac{\gamma}{\dot{Q}_{s p}} F\left(\xi, \xi_{s}, T_{a d}\right) \quad \text { for } \xi z \xi_{s}
$$

$$
\text { while } \quad Y_{s}=0, \quad \xi<\xi_{s}
$$

where $\gamma$ is the straining rate and $F$ is a function obtained by integrating eqn. (1b) over an appropriate streamline (from 0 to $\Psi_{\nu}$ ). Its exact form is not required for the present work, which focuses on optically thin flames. Note that eqn. (3b) has been derived from a simplification of the oxidation process according to which the oxidation rate in the fuel lean side $\left(\xi<\xi_{2}\right)$ is so fast that soot oxidizes instantaneously there. This situation is applied as long as the (maximum) laminar flamelet temperature (at stoichiometric condition) is greater than a certain value, about[3] $1500 \mathrm{~K}$; otherwise, soot can not be further oxidized and is emitted as smoke.

By using eqn. (3) and the function of temperature in terms of mixture fraction, $T(\xi)$, one obtains from eqn. (2) an average volumetric radiation loss: 


$$
\begin{aligned}
& \dot{q}_{s}^{\prime \prime \prime}=3.26 \cdot 10^{-4} \frac{P}{R \rho_{s}} Y T^{4}= \\
& =3.26 \cdot 10^{-4} \frac{P}{R \rho_{s}} \iint \frac{\gamma}{\dot{Q}_{s p}} F\left(\xi, \xi, T_{L, \max }\right) T^{4}(\xi) P(\xi, \gamma) d \xi d \gamma
\end{aligned}
$$

wherein $\mathrm{P}(\xi, \gamma)$ in the joint pdf between the mixture fraction, $\xi$, and the straining rate, $\gamma$. Next, we use the common approximation that mixture fraction and straining rate are statistically independent so that eqn. (4a) becomes:

$$
\dot{q}_{s}^{\prime \prime \prime}=3.26 \cdot 10^{-4} \frac{P}{R \rho_{s}} \frac{\bar{\gamma}}{\dot{Q}_{s p} 0} \int_{0}^{1} F T^{4} P(\xi) d \xi
$$

It is this relationship that is applied in the energy balance of the present integral combustion model along the jet axis:

$$
\frac{d}{d z} \int h d \psi=-\int_{0}^{\infty} \dot{q}_{s}^{\prime \prime \prime} 2 \pi r d r
$$

where $h$ is the total enthalpy of the product of combustion (i.e. chemical +convective) and $\Psi$ is the stream function for the mean axial jet velocity. In this form, eqn. (5a) is difficult to solve owing to the iterations needed to obtain the flame temperature for the laminar flamelets or for the turbulent flow.

In the spirit of the integral model (and to save computational time), we make the approximation that the laminar flamelet temperature at a given height from the source is uniformly reduced by a constant fraction $\alpha$ of its adiabatic value, namely:

$$
T_{L}(\xi)-T_{\infty}=\alpha\left(T_{L, \alpha d}(\xi)-T_{\nu}\right)
$$

while the dependence of species concentrations on $\xi$ remains the same independent of the radiation losses as long as the maximum flamelet temperature is greater than 1,500 $\mathrm{K}$ (this approximation is supported by experiments[6]). With these approximations, the energy conservation equation (5a) becomes:

$$
\frac{d}{d z}\left[(1-\alpha) \dot{Q}_{c h}(z)\right]=\int_{0}^{\infty} \dot{q}_{s}^{\prime \prime \prime} 2 \pi r d r
$$

where $\dot{Q}_{c h}(z)$ is the chemical (heat of formation flux) heat release at height $z$ and $(1-\alpha)$ can also be identified to be equal to the radiant fraction up to height $z$ :

$$
1-\alpha=\chi_{R}(z)=\frac{\dot{Q}_{R}(z)}{\dot{Q}_{c h}(z)}
$$

where $\dot{Q}_{R}(z)$ is the radiation losses from the flame over a length extending from the source to a given height $\mathrm{z}$. Even in the simplified form, eqn. (5c) requires iterations over the whole 
field in order to obtain the value of $\alpha$ owing to the complicating dependence of radiant losses on temperature as indicated in eqn. (4b). A further simplification of eqn. (5c) has been obtained based on overall radiation correlation[3] and the specific form of radiations losses (eqn. (4b)):

$$
\int_{0}^{\infty} \dot{q}_{s}^{\prime \prime \prime} 2 \pi r d r=\frac{C \cdot \sigma T_{\alpha d}^{A}}{(1-\alpha)^{3} \dot{Q}_{s p}} \int_{0}^{\phi_{t}} P\left(\xi_{s}, \bar{\xi}\right) \frac{d \Psi}{\bar{\rho} \bar{u}}
$$

The following remarks are made concerning the derivation of eqn. (6a):

1. Most of the contributions to radiation losses originates for values of $\xi \approx \xi_{8}+\epsilon$ near the stoichiometric value so that:

$$
F T^{4}-\text { delta function } \sim \delta\left(\xi-\xi_{g}\right)
$$

2. The average strain rate $\bar{\gamma}$ (which can be identified with the Kolmogorov strain rate) is nearly independent of location or total heat release rate for buoyant flames[3]; therefore it is assumed to have a constant value.

3. The reduction of the term $\mathrm{FT}^{4}$ due to radiation losses is proportional to $1 / \chi_{\mathrm{R}}{ }^{3}$. This dependence has been deduced from global correlations on radiation[3].

4. The constant $\mathrm{C}$ incorporates all the physical constants and the constants associated with the assumptions $1,2,3$. It has to be determined from experiments.

5. Comparison with experiments demonstrates the applicability of the approximations used to derive eqn. (6a). More importantly, the value of the constant $\mathrm{C}$ should be independent of the fuel, assuming that the present approximations are adequate.

Finally, with these approximations, the energy balance equation $(5 \mathrm{c})$ becomes:

$$
\frac{d}{d z}\left[(1-\alpha) \dot{Q}_{c h}(Z)\right]=\frac{C \sigma T_{\alpha d}^{4}}{(1-\alpha)^{3} \dot{Q}_{s p}} \int_{0}^{\phi_{t}} P\left(\xi_{s}, \bar{\xi}\right) \frac{d \Psi}{\bar{\rho} \bar{u}}
$$

We may also characterize the radiation modelling in eqn. (7) in the following way:

1). All radiation originates from soot that is near the instantaneous maximum flamelet temperature;

2). This temperature, however, decreases owing to radiant losses as the radiation fraction increases.

Equations (7) is singular near the flame base where $(1-\alpha) \rightarrow 0$. We can not model the flow conditions near the flame base at this moment. Fortunately because we have used integrated value over the entire length of the flame, this effect has been eliminated.

\section{COMPARISON OF PREDICTION WITH EXPERIMENTS}

Eqn. (7) together with the remaining conservation equations (i.e., momentum, mixture fraction), as well as with the flamelet combustion model, form a well-behaved system of equations which have been applied to several cases. Results and comparison for the radiation 
distribution are presented in this section for turbulent buoyant jets and pool fires of various fuels.

Prediction of heat release rate from the integral combustion model and comparison with experimental data[8] for a propane $\left(\mathrm{C}_{3} \mathrm{H}_{8}\right)$ buoyant jet flame are illustrated in Figure 2. The abscissa is the ratio between the height from the nozzle to two times $\mathrm{L}_{0.5}\left(\mathrm{~L}_{0.5}\right.$ is the distance at which onehalf of total heat release occurs) and the ordinate is the scaled heat release rate, i.e. the ratio of the heat release rate at that height, $\dot{Q}_{\mathrm{ch}}(\mathrm{z})$ to the total heat rate, $\dot{Q}_{t h}$. The burner diameter used in the experiment was $12.7 \mathrm{~mm}$ and the jet flame theoretical heat release rate was $30.8 \mathrm{~kW}$. The comparison in Figure 2 shows very good agreement between prediction from the model and the experimental data for heat release rate.

The constant $\mathrm{C}$, in the energy balance eqn. (7), was determined by requiring that at the end of combustion the radiation fraction $(1-\alpha)$ is equal to the total radiation fraction for the fuel, which is known if the smoke-point heat release rate, $\dot{Q}_{\mathrm{sp}}$, is known[3] (for propane,

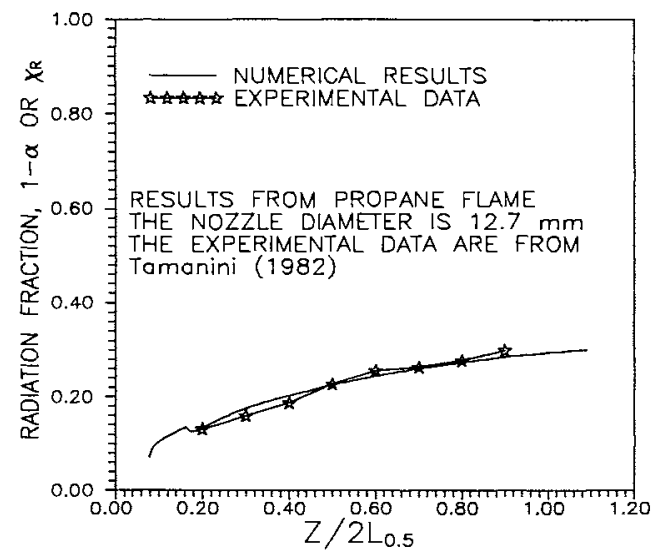

Figure 3a Radiation fraction at a given height plotted versus the height normalized by twice the length, $\mathrm{L}_{0.5}$, over which half of the combustion has been completed

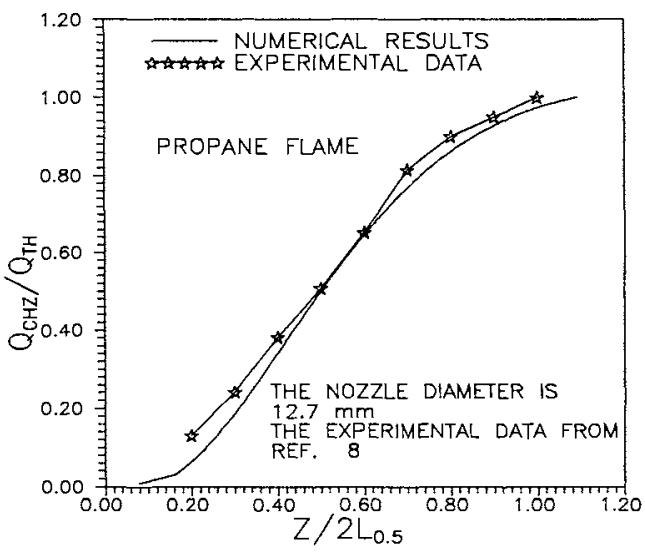

Figure 2 Chemical heat release rate normalized by the total heat release rate versus distance from the nozzle exit, normalized by twice the length, $\mathrm{L}_{0.5}$, over which half of the combustion has been completed. constant was found to be, $C=0.36$ $\underline{W} / \mathrm{s}$ if $\dot{Q}_{\mathrm{sp}}$ is expressed in $\mathrm{W}$. By using this value, the calculated radiation fraction, $\chi_{\mathrm{R}}$ vs. the scaled height from the nozzle is plotted in Figure $3 \mathrm{a}$ while the scaled radiation per unit length of the flame is illustrated in Figures $3 b$ and 3c. All the experimental data were obtained by Tamanini[8]. The close behavior between the prediction and the experiment, shown in Figure $3 \mathrm{a}$ reinforces the validity of this approach. The prediction does give the right trend of radiation loss distribution (Figures $3 b$ and 3c) though the difference with experiments is fairly large. It can be noted (may not be obvious) that the area under the curves from the prediction (Figure $3 b$ ) 
is the same as that from the experiment.

Finally, Figure 4 illustrates the maximum laminar flamelet temperature variation as a function of the height from the flame base. The result shows decreasing laminar flame temperature as radiation losses increase. Near the flame tip $(\sim 1 \mathrm{~m})$, the laminar flamelet temperature is about $1680 \mathrm{~K}$ indicating that no extinguishment of the flame due to radiation loss occurs. Therefore, soot is probably completely oxidized inside the flames, which agrees with experiments[2] that little smoke is emitted from propane flames.

The same constant $\underline{C}(=0.36$ $\mathrm{W} / \mathrm{s}$ ), can be used to calculate the combustion and radiation of another fuel, propylene, if the smoke-point heat release rate, $\dot{Q}_{\mathrm{xp}}$, is known (see eqn. (7)). We have used this approach to predict the radiation of propylene $\left(\mathrm{C}_{3} \mathrm{H}_{6}, \dot{\mathrm{Q}}_{\mathrm{sp}}=46.3 \mathrm{~W}\right)$. The results are shown in Figures $5(\mathrm{a}-\mathrm{c})$. In Figure 5a, the prediction of radiation fraction is plotted against the height from the flame; the total radiation fraction is about 0.46 which is close to 0.44 as measured in an experiment[3]. Figure $5 b$ shows a comparison of non-dimensional radiation distribution between the prediction and experiments[9]. These results show that one can use the smoke-point heat release rate to predict total radiation and radiation distribution from buoyant jet flames. For completeness, the maximum laminar flamelet temperature for propylene flames is shown in Figure 5c. In contrast to the propane flames, Figure 4, the temperature drops below $1,500 \mathrm{~K}$ at a height at $0.60 \mathrm{~m}$; such a low temperature will result in flame quenching, copious smoke yield and incompleteness of combustion, which might explain the higher calculated value of total radiation fraction, 0.46 , relative to the numerical value of 0.44 . Incompleteness of combustion will be addressed as the present model is carefully evolved.

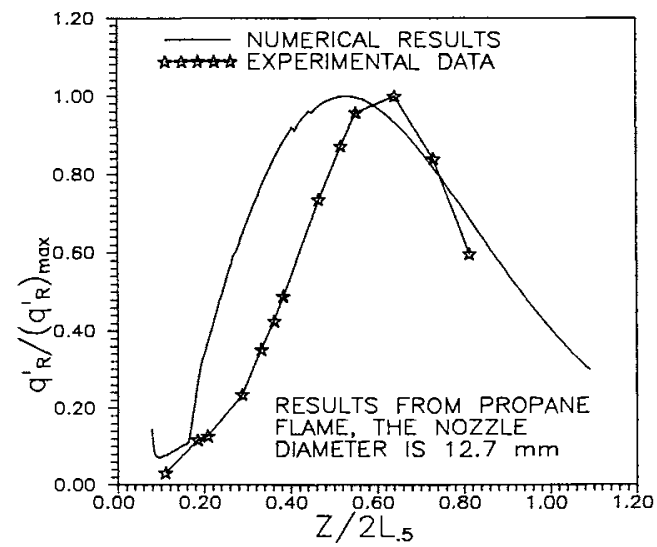

Figure 3b Radiation distribution per unit height versus normalized distance from the nozzle exit (cf. Fig. 3a). The experimental data are from Tamanini[8]

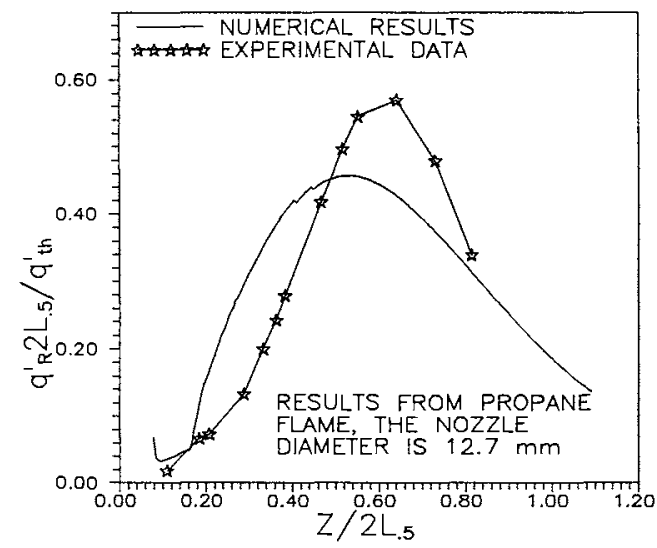

Figure 3c Same data as in Figure 3b, except that the radiation per unit height is normalized by its maximum value. The experimental data are from Tamanini[8] 


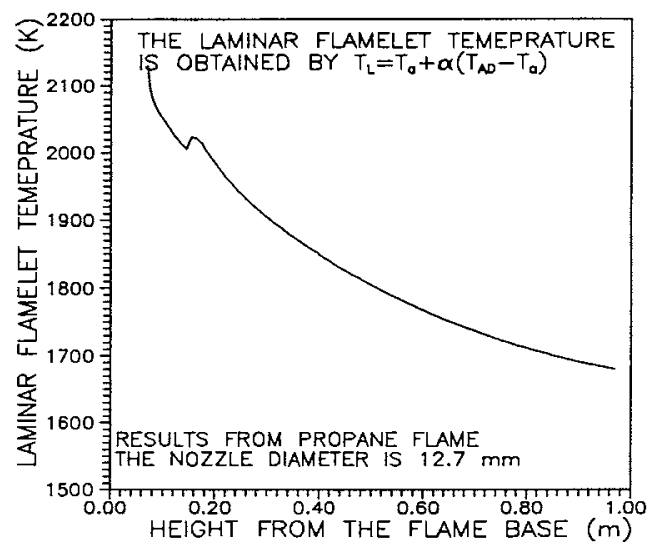

Figure 4 Reduction of laminar flamelet temperature with height owing to radiation losses for the use of Figure 2
The above discussions and results presented in Figures $5 \mathrm{a}$ and $5 \mathrm{~b}$ have shown that use of a single constant (representing collective physical parameters) is feasible for radiation modeling. This also points out that the integral model has accurately included the essential physics for buoyant jet flames.

A final demonstration that the present model could be extended to combustion and radiation in pool fires is shown in Figures $6 \mathrm{a}$, and $6 \mathrm{~b}$, for a propane fire $0.38 \mathrm{~m}$ in diameter. The same conservation equations were used for a turbulent buoyant jet flame including the constant, $\mathrm{C}(=0.36 \mathrm{~W} / \mathrm{s})$ for radiation of $\mathrm{C}_{3} \mathrm{H}_{8}$. The following modifications were made:

1) an entrainment rate correlation for pool fires was used[10] and

2) the fluctuations were represented by the relationship:

$$
\frac{\overline{\xi^{\prime 2}}}{\bar{\xi}(1-\bar{\xi})}=0.11
$$

instead of eqn (1a) because eqn. (8) was shown[1] to be the one applicable for pool fires. This expression represents a level of fluctuations, $\left(\xi^{2}\right)^{1 / 2} / \bar{\xi}$, which for values of $\bar{\xi}$ close to 1 is smaller than what eqn. (1a) represents; this behavior is consistent with measurements of fluctuations in pool fires[11].

The agreement between predictions and experiments as shown in Figures $6 \mathrm{a}$ and $6 \mathrm{~b}$ is more encouraging than should be expected, because the elliptic flow field near the pool base is not modeled in the present approach. In faimess to the present work, it should be pointed out that measurements of $\chi_{\mathrm{R}}$ in pool fires in Tamanini's [8] experiments (wherein he used a "catcher-quencher" to

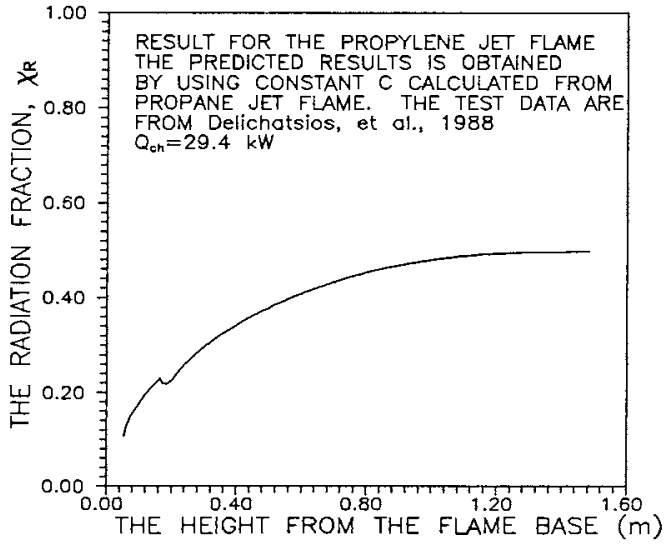

Figure 5a Prediction of radiation fraction versus height for propylene turbulent buoyant jet flame. No experiments are available except for the total radiation fraction 
measure heat release rates) have large errors $( \pm 50 \%)$ near the pool base $(\leq .20 \mathrm{~m}$ ) because a) the width of the slit radiometer used to measure the radiation per unit height was $54 \mathrm{~mm}$ and b) the "catcher-quencher" might have influenced the combustion. A more detailed examination of the shortcoming of pool fire modeling is currently being undertaken.

\section{CONCLUSIONS}

It was shown how a phenomenological soot formation model based on laminar smoke-point height (see eqns. (1b) and (1c)) can be applied in turbulent combustion to

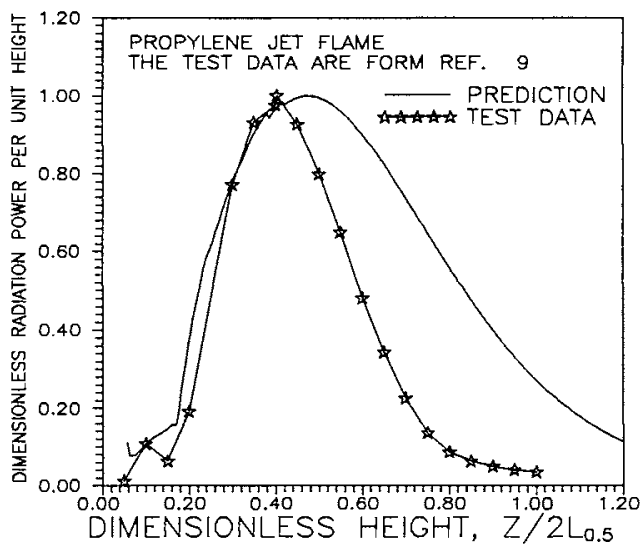

Figure 5b Radiation distribution per unit height versus height for a propylene turbulent buoyant jet flame

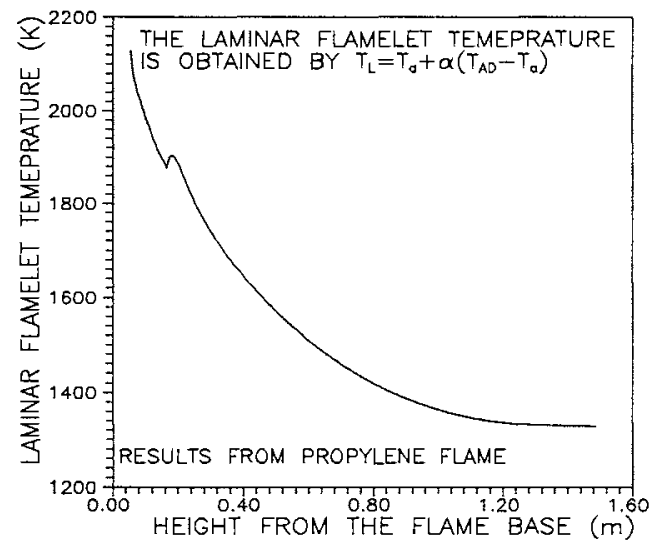

Figure 5c Reduction of laminar flamelet temperature vs. height owing to radiation loss for the case of Figure 5a

increases. predict soot concentration and flame radiation. As a first step this soot and radiation model was applied to optically thin flames to derive an energy equation whose integral expression is given by eqn. (7). Comparison of predictions with radiation measurements in turbulent buoyant jet flames (Figures 3a, 3b, 3c and Figures $5 \mathrm{a}, 5 \mathrm{~b}$ ) for different fuels provided a partial validation to the present model. For pool fires, application of the model provides encouraging results (Figure $6 a, 6 b$ ) but it also points out a need for a) further examination of the flow near the base of the pool fire and b) relaxation of the optically thin flame approximation as the pool size 


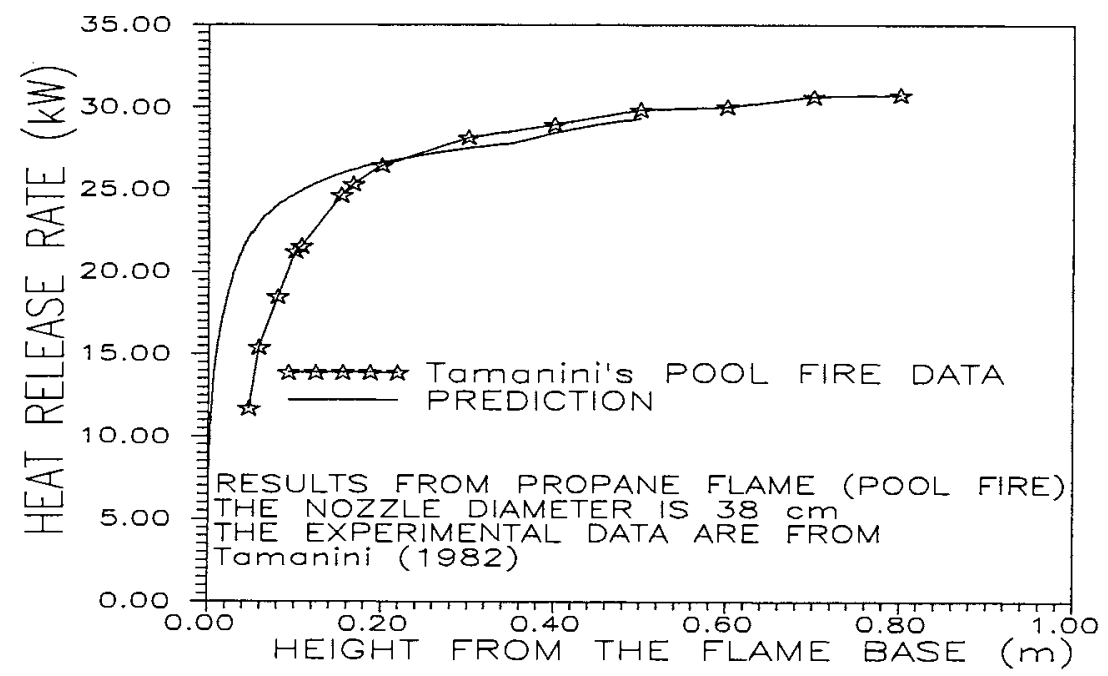

Figure 6a Chemical heat release rate versus distance from the base of a propane pool fire 0.38 in diameter

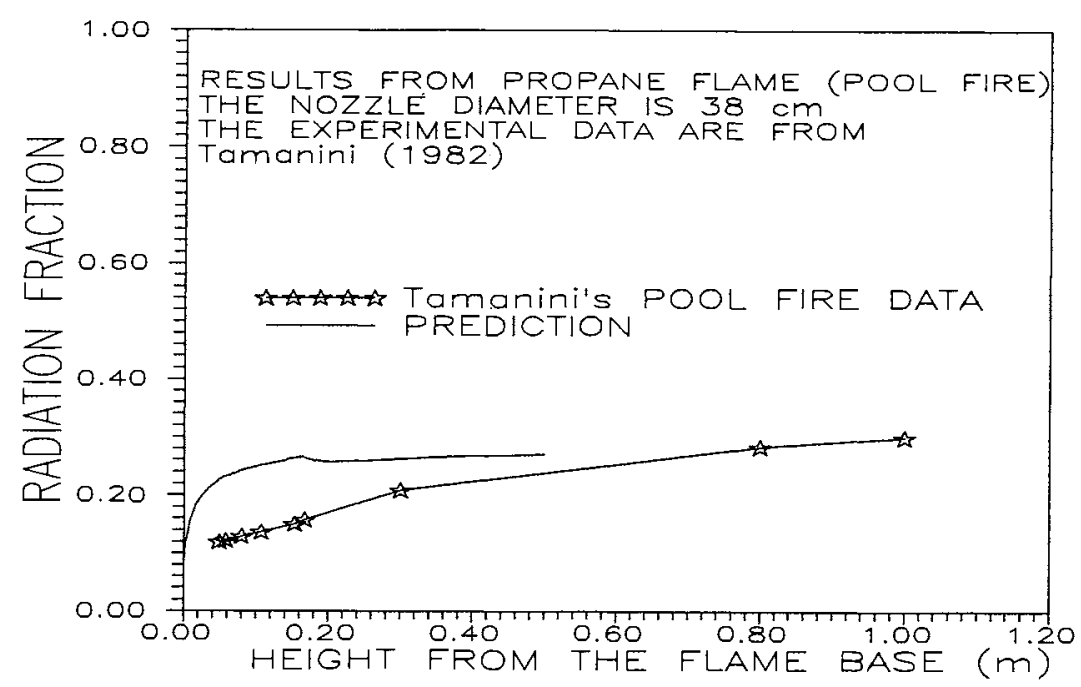

Figure $6 \mathrm{~b}$ Radiation friction at a given height plotted versus the height from the base of a propane pool fire $0.38 \mathrm{~m}$ in diameter 


\section{REFERENCES}

1. Delichatsios, M.A. and Mathews, M.K., "A New Simplified PDF Method for Calculating Major Species Concentrations and Burning in Turbulent Fires," 2nd. Int'1 Symp. on Fire Safety Science, pp. 149-158, 1989.

2. Tamanini, F., "An Integral Model of Turbulent Fire Plumes," 18th (Int'1) Symp. on Comb., The Combustion Institute, pp. 1081-1089, 1981.

3. Delichatsios, M.A., Orloff, L. and Delichatsios, M.M., "The Effect of Fuel Sooting Tendency and the Flow on Flame Radiation in Luminous Turbulent Jet Flames," Comb. Sci. and Tech., 84, pp. 199-215, 1992.

4. Chen, Y., Motevalli, V. and Delichatsios, M.A., "An Integral Turbulent Radiation and Combustion Model for Diffusion Flames,", Proc. of the Eastern and Central Section Meeting of the Combustion Institute, pp. 51-55, 1993.

5. Chatwin, P.C. and Sullivan, P.J., "A Simple and Unifying Physical Interpretation of Scalar Fluctuation Measurements from Many Turbulent Shear Flows, J. Fluid Mech., 212, pp. 533-555, 1990.

6. Kolyu, O.V., Kounalakis, M.E., Sivathanu, Y.R. and Faeth, G.M., "Carbon Monoxide and Radiation Emissions of Turbulent Diffusion Flames," NIST Technical Report, Grant No. 60NANBD0833, 1992.

7. Delichatsios, M.A., "A Phenomenological Model for Soot Formation in Laminar Flames", accepted for Publication in Comb. Sci. and Tech., 1993.

8. Tamanini, F., "Direct Measurements of the Longitudinal Variation of Buming Rate and Product Yield in Turbulent Diffusion Flame," FMRC Technical Report No. J.I. OFON4,BU, 1982.

9. Delichatsios, M.A., Markstein, G.H., Orloff, L. and de Ris, J., "Turbulent Flow Characterization and Radiation From Gaseous Fuel Jets," Gas Research Institute, GRI-88/0100, 1988.

10. Delichatsios, M.A., "Air Entrainment into Buoyant Jet Flames and Pool Fires," Comb. Flame, 70, pp. 33-46, 1987.

11. Gengebre, E., Cambray, P., Karmed, D. and Bellet, J.C., "Turbulent Diffusion Flmaes with Large Buoyant Effects," Comb. Sci. Tech., 41, pp. 55-67, 1984. 